\title{
NdFeCo TRILAYER AMORPHOUS FILMS WITH HIGH KERR ROTATION AT SHORT WAVELENGTHS
}

\author{
H. liyori and S. Takayama \\ IBM Research, Tokyo Research Laboratory, IBM Japan Ltd., 1623-14, Shimo-tsuruma, \\ Yamato, Kanagawa 242, Japan
}

\begin{abstract}
Magneto-optical recording materials with high remanent Kerr rotation at short wavelengths were successfully obtained in a trilayer form. A NdFeCo amorphous film with in-plane magnetization, but with a large saturation Kerr rotation angle at short wavelengths, was sandwiched between $\mathrm{TbFeCo}$ amorphous films with strong perpendicular anisotropy and high coercivity. The trilayer film, $\mathrm{Tb}_{20} \mathrm{Fe}_{74} \mathrm{Co}_{6}(10 \mathrm{~nm}) / \mathrm{Nd}_{16} \mathrm{Fe}_{57} \mathrm{Co}_{27}(7.5 \mathrm{~nm}) / \mathrm{Tb}_{20} \mathrm{Fe}_{74} \mathrm{Co}_{6}(100 \mathrm{~nm})$, shows a remanent Kerr rotation angle $\theta_{\mathrm{Kr}}=0.32^{\circ}$ at a wavelength of $400 \mathrm{~nm}$, coercivity $\mathrm{H}_{\mathrm{c}}=2.4 \mathrm{kOe}$, and a good square loop $\left(\theta_{\mathrm{Kr}} / \theta_{\mathrm{Ks}} \sim 1\right)$.
\end{abstract}

KEYWORDS: TRILAYER FILM, NdFeCo, SHORT WAVELENGTHS, EXCHANGE COUPLING

\section{INTRODUCTION}

Magneto-optical (M.O.) materials with high Kerr rotation at short wavelengths have recently received much attention for their potential use in future highdensity magneto-optical recording $[1-6]$. We previously reported that we had successfully obtained such an M.O. medium by employing a trilayer NdCo amorphous alloy film, which showed good squareness in its Kerr hysteresis loops and a high coercive force $\mathrm{H}_{c}(2$ kOe) $[7,8]$. In these layered structure, a NdCo amorphous film with an in-plane magnetization are sandwiched between $\mathrm{TbFeCo}$ films with strong perpendicular anisotropy and high coercivity.

In this report, we describe our subsequent attempt to obtain a larger remanent Kerr rotation angle by employing a $\mathrm{NdFeCo}$ film as a middle layer. We used $\mathrm{NdFeCo}$ because it has a larger magneto-optical effect than $\mathrm{NdCo}$ at short wavelengths of around $400 \mathrm{~nm}$. $[3-7]$. One of key features of the proposed trilayer structure is that the middle layer ( $\mathrm{NdFeCo})$ is thick enough to give rise to a high Kerr rotation, but thin enough to reorient the magnetization in a direction perpendicular to the film surface through the whole film thickness by exchange coupling.

As a result, we successfully obtained a remanent Kerr rotation angle of 0.32 degree at a short wavelength of $400 \mathrm{~nm}$. with a good square loop $\left(\theta_{\mathrm{Kr}} / \theta_{\mathrm{Ks}} \sim 1\right)$ and a coercivity of $2.4 \mathrm{kOe}$. The figure of merit, $\left(\sqrt{\mathrm{R}} \theta_{\mathrm{k}}\right)$, of this trilayer film is 0.21 at a wavelength of $400 \mathrm{~nm}$, which is larger than that of the previous NdCo trilayer film [8].

\section{EXPERIMENT}

Trilayer NdFeCo films ( $\mathrm{TbFeCo} / \mathrm{NdFeCo} / \mathrm{TbFeCo})$ were prepared on glass substrates by using d.c. magnetron sputtering without breaking a vacuum during the multilayer process. The background vacuum before sputtering was less than $1.0 \times 10^{-6}$ Torr. Ar pressure during sputtering was $5.0 \times 10^{-3}$ Torr. We employed targets for $\mathrm{NdFeCo}$, Nd sheets on the $\mathrm{Fe}-\mathrm{Co}$ alloy target, and for $\mathrm{TbFeCo}$, Tb sheets on the $\mathrm{Fe}$-Co alloy target. The magnetic properties of the trilayer films were measured with a vibrating sample magnetometer (VSM) in fields up to about $20 \mathrm{kOe}$, and the magnetooptical properties were obtained with a Kerr loop tracer in fields up to $15 \mathrm{kOe}$. The atomic compositions of $\mathrm{NdFeCo}$ and $\mathrm{TbFeCo}$ were measured by using an inductively coupled plasma spectrometer (ICP). The anisotropy constant for $\mathrm{NdFeCo}$ was obtained from the torque curves, measured in a field of about $19 \mathrm{kOe}$, and that for TbFeCo was measured by the Miyajima method [9] in fields up to $19 \mathrm{kOe}$.

\section{RESULTS AND DISCUSSION}

The schematic cross-section of the trilayer films investigated is shown in the insert of Fig. 1. The atomic composition of the first and third ( $\mathrm{TbFeCo}$ ) layers is Tb $20 \%, \mathrm{Fe} 74 \%$, and $\mathrm{Co} 6 \%$, while that of the second ( $\mathrm{NdFeCo}$ ) layer is $\mathrm{Nd} 16 \%$, Fe $57 \%$, and Co $27 \%$. The $\mathrm{Tb}_{20} \mathrm{Fe}_{74} \mathrm{Co}_{6}$ amorphous films shows coercivity $\mathrm{H}_{\mathrm{c}}=$ $9.8 \mathrm{kOe}$, saturation magnetization $\mathrm{M}_{\mathrm{S}}=84 \mathrm{emu} / \mathrm{cc}$, effective perpendicular anisotropy $\mathrm{K}=5.3 \times 10^{6} \mathrm{erg} / \mathrm{cc}$, and Curie temperature $\mathrm{T}_{\mathrm{c}}=205^{\circ} \mathrm{C}$, while the $\mathrm{Nd}_{16} \mathrm{Fe}_{57} \mathrm{Co}_{27}$ amorphous films give figures of $\mathrm{M}_{\mathrm{S}}=1.1 \times 10^{3} \mathrm{emu} / \mathrm{cc}$, and $\mathrm{K}=-4.4 \times 10^{6} \mathrm{erg} / \mathrm{cc}$. To optimize the trilayer structure, the thickness of the first and third (TbFeCo) layers were fixed at 10 and $100 \mathrm{~nm}$, respectively. The thickness of the second ( $\mathrm{NdFeCo}$ ) layer was varied from 


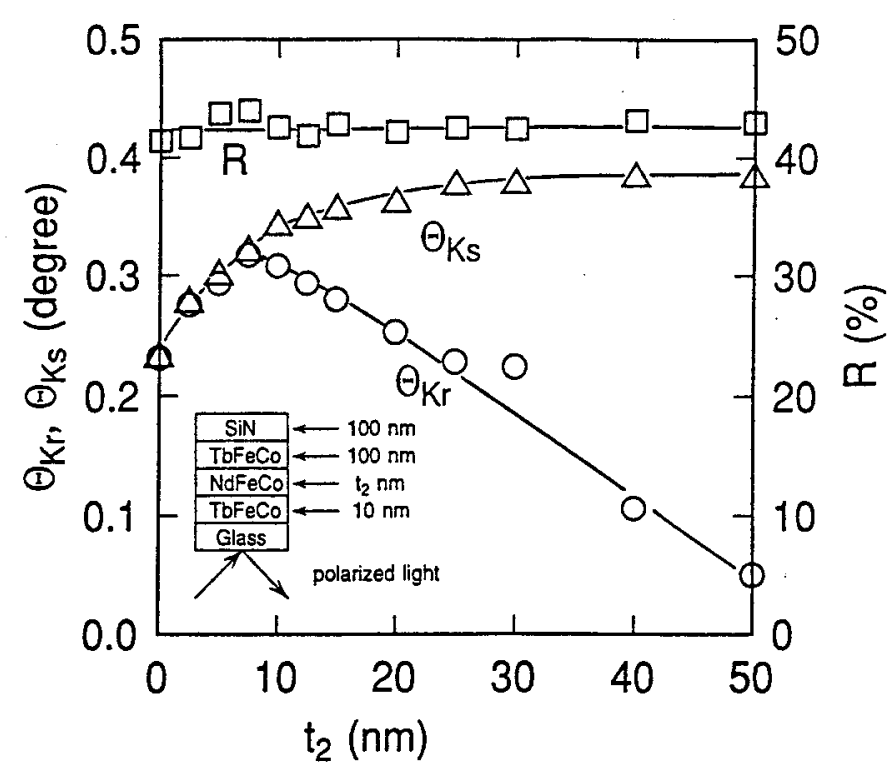

Fig. 1 Remanent Kerr rotation angle $\theta_{\mathrm{Kr}}$, saturation Kerr rotation angle $\theta_{\mathrm{Ks}}$, and reflectivity $R$ as a function of the second layer thickness $(\mathrm{NdFeCo}) t_{2}$, measured at $400 \mathrm{~nm}$

0 to $50 \mathrm{~nm}$. Fig. 1 shows the remanent Kerr rotation angle $\theta_{\mathrm{Kr}}$, the saturation $\mathrm{Kerr}$ rotation angle $\theta_{\mathrm{Ks}}$, and the reflectivity $\mathrm{R}$, measured at $\lambda=400 \mathrm{~nm}$ through a glass substrate, as a function of the second $(\mathrm{NdFeCo})$ layer thickness $\left(\mathrm{t}_{2}\right)$. As the thickness of the second layer increases, the remanent Kerr rotation angle increases, reaching a peak around the second layer thickness of $7.5 \mathrm{~nm}$. With a further increase in the second layer thickness, $\theta_{\mathrm{Kr}}$ starts to decrease. The initial increase in the remanent Kerr rotation angle is associated with an increase in the depth of effective light penetration into the second layer $(\mathrm{NdFeCo})$, whose magnetization is effectively reoriented by the first and third layers in the direction normal to the film plane. All the above Kerr loops and magnetization curves show good squareness. A decrease in the remanent Kerr rotation angle above the second layer thickness of $7.5 \mathrm{~nm}$ is due to an increase in the in-plane magnetization of the second $(\mathrm{NdFeCo})$ layer. This means that the first and the third $(\mathrm{TbFeCo})$ layers cannot reorient the magnetization of the second $(\mathrm{NdFeCo})$ layer sufficiently in the direction normal to the film plane. On the other hand, the saturation Kerr rotation angle $\theta_{\mathrm{Ks}}$ becomes larger as the thickness of the second layer increases from $t_{2}=0$ to $20 \mathrm{~nm}$. Above the second layer's thickness of $25 \mathrm{~nm}$, the saturation Kerr rotation angle becomes almost constant, $\theta_{\mathrm{Ks}}=0.38^{\circ}$. Since the total saturation Kerr rotation angle measured is the weighted average of the portions of the top, middle, and bottom layers that the light reaches, as stated above, this result indicates that the penetration depth in the second layer is about $20 \mathrm{~nm}$. Note that the thickness of the second layer when the remanent Kerr rotation angle is at a maximum is less than that of the NdCo trilayer film previously reported [9], because a $\mathrm{NdFeCo}$ amorphous thin film has large demagnetization field as a result of $\mathrm{NdFeCo}$ having a higher magnetization than NdCo. The reflectivity R of trilayer films at $\lambda=400 \mathrm{~nm}$ shows an almost constant value $(42.7 \%)$, which means that the multi-reflection of the second layer does not contribute to the enhancement of the Kerr rotation angle, because it reduces the reflectivity.

Representative Kerr hysteresis loops at a wavelength of $400 \mathrm{~nm}$ and magnetization curves for the samples at $\mathrm{t}_{2}=7.5$ and $25 \mathrm{~nm}$ in Fig. 1 are shown in Figs. 2(a) and 2(b), respectively. Both curves in Figs. 2(a) show good squareness $\left(\mathrm{M}_{\mathrm{r}} / \mathrm{M}_{\mathrm{S}} \sim 1\right)$ and high coercivity $\left(\mathrm{H}_{\mathrm{c}}=2.4 \mathrm{kOe}\right)$. The direction of magnetization is reversed abruptly. This means that the each layer in a trilayer film is well coupled by exchange coupling. On the other hand, the Kerr loop and the magnetization curve in Figs. 2(b) show poor squareness, because of the relative increase in the in-plane magnetization of $\mathrm{NdFeCo}$ in the second ( $\mathrm{NdFeCo}$ ) layer of the the trilayer film. Both curves in Figs. 2(b) have a shoulder when the magnetization of the film is changed. These shoulders are caused by the state in which the magnetization of the first layer is antiparallel to the magnetization of the third layer [12].

A typical torque curve of trilayer films is shown in Fig. 3. The sample is the same as that shown in Fig. 2(a). This curve also has a shoulder when the sign of
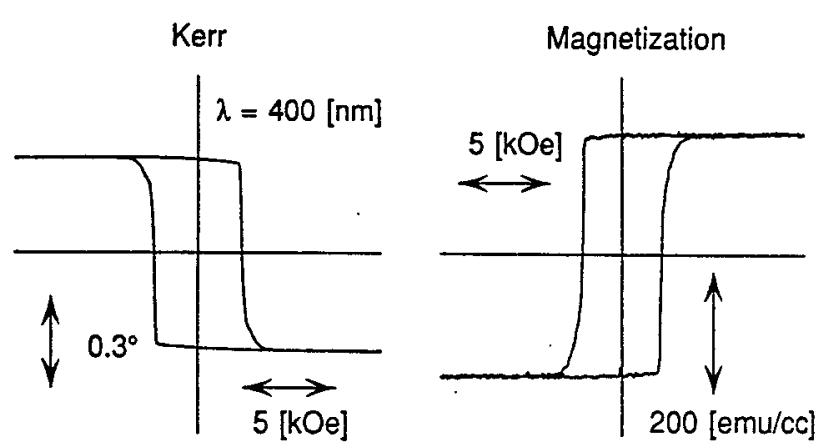

(a) TbFeCo $(10 \mathrm{~nm}) / \mathrm{NdFeCo}(7.5 \mathrm{~nm}) / \mathrm{TbFeCo}(100 \mathrm{~nm})$
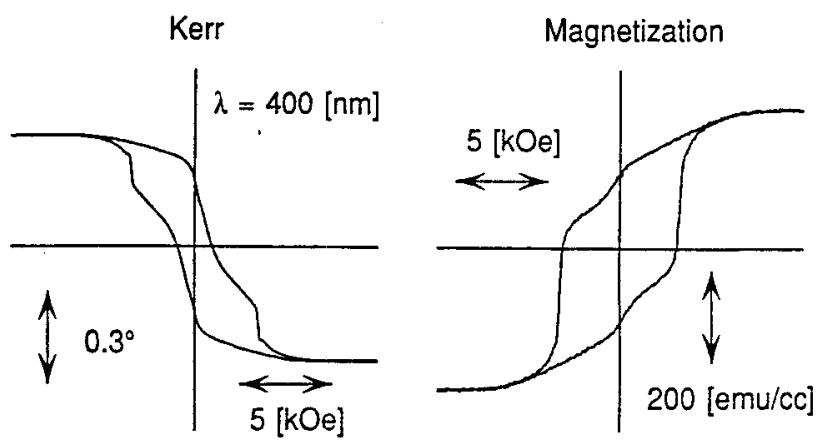

(b) TbFeCo $(10 \mathrm{~nm}) / \mathrm{NdFeCo}(25 \mathrm{~nm}) / \mathrm{TbFeCo}(100 \mathrm{~nm})$

Fig. 2 Kerr hysteresis loops and magnetization curves for trilayer films 


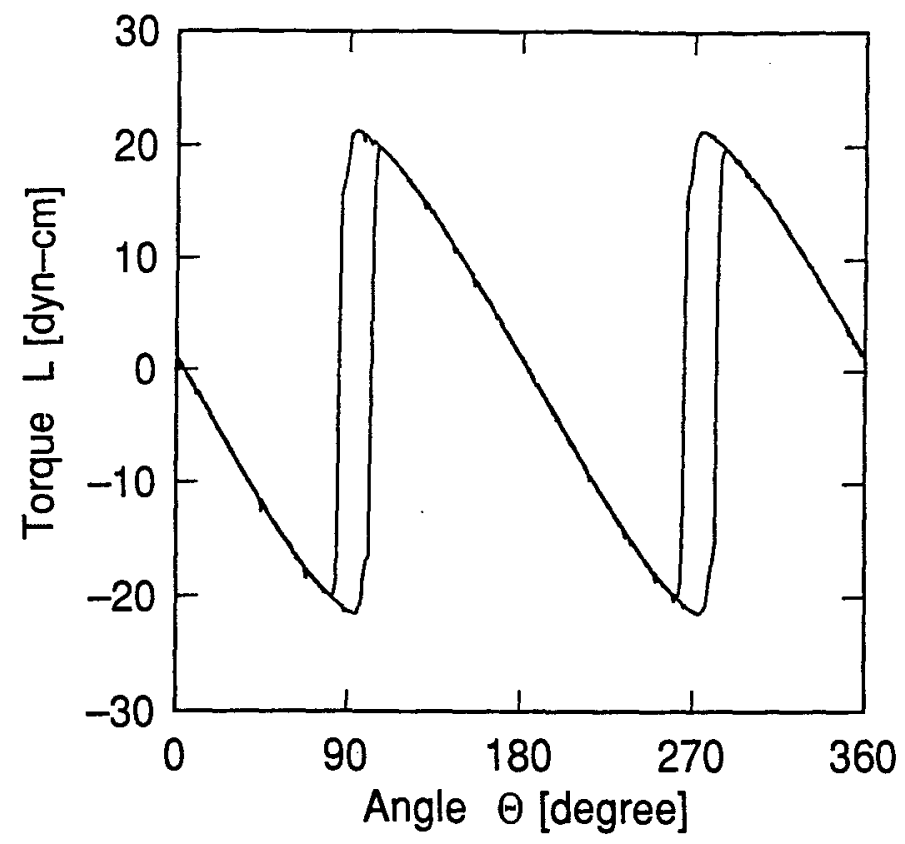

Fig. 3 A torque curve for a $\mathrm{Tb}_{20} \mathrm{Fe}_{74} \mathrm{Co}_{6}(10 \mathrm{~nm}) / \mathrm{Nd}_{16} \mathrm{Fe}_{57} \mathrm{Co}_{27}$ $(7.5 \mathrm{~nm}) / \mathrm{Tb}_{20} \mathrm{Fe}_{74} \mathrm{Co}_{6}(100 \mathrm{~nm})$ trilayer film, measured in a field of $19 \mathrm{kOe}$

the torque is reversed. These shoulders most likely are created when the magnetization direction of the first layer is antiparallel to that of the third layer [12].

Figure 4 shows the wavelength dependence of the Kerr rotation $\theta_{\mathrm{Kr}}$ and reflectivity $R$ for samples of a $\mathrm{Tb}_{20} \mathrm{Fe}_{74} \mathrm{Co}_{6}(10 \mathrm{~nm}) / \mathrm{Nd}_{16} \mathrm{Fe}_{57} \mathrm{Co}_{27}(7.5 \mathrm{~nm}) /$

$\mathrm{Tb}_{20} \mathrm{Fe}_{74} \mathrm{Co}_{6}(100 \mathrm{~nm})$ trilayer film $(\mathrm{T}-\mathrm{NdFeCo})$ and of a $\quad \mathrm{Tb}_{23} \mathrm{Fe}_{72} \mathrm{Co}_{5}(10 \mathrm{~nm}) / \mathrm{Nd}_{20} \mathrm{Co}_{80}(10 \mathrm{~nm}) /$ $\mathrm{Tb}_{23} \mathrm{Fe}_{72} \mathrm{Co}(100 \mathrm{~nm})$ trilayer film(T-NdCo). The dependence of $a \mathrm{~Tb}_{20} \mathrm{Fe}_{74} \mathrm{Co}_{6}$ single film with the same composition as the first and third layers of $\mathrm{NdFeCo}$

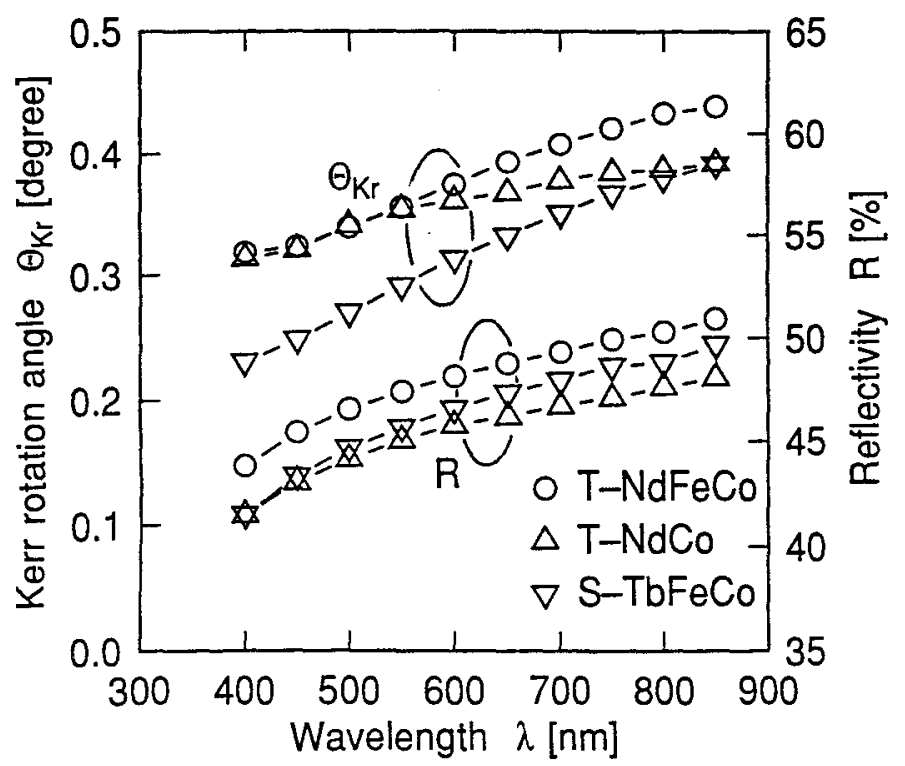

Fig. 4 Light wavelength dependence of remanent Kerr rotation $\theta_{\mathrm{Kr}}$ and reflectivity $\mathrm{R}$ trilayer films is also shown for comparison. Note that the remanent Kerr rotation angle of the present $\mathrm{NdFeCo}$ trilayer film ( $\mathrm{T}-\mathrm{NdFeCo}$ ) is larger than that of the former NdCo trilayer film (T-NdCo) above a wavelength of $600 \mathrm{~nm}$, and larger than that of a TbFeCo single-layer film throughout the measured range. This most likely reflects the fact that a $\mathrm{NdFeCo}$ film has a larger intrinsic magneto-optical effect than a TbFeCo film and a NdCo film throughout the measured wavelength region. The $\mathrm{NdFeCo}$ trilayer film (T-NdFeCo) has almost the same remanent Kerr rotation angle as the NdCo trilayer film (T-NdCo) at wavelengths from 400 to $550 \mathrm{~nm}$, even though the thickness of the second layer $(7.5 \mathrm{~nm})$ is thinner than that of the NdCo trilayer film $(10 \mathrm{~nm})$. This results from the fact that the second layer (NdFeCo) has a higher saturation magnetization and a higher saturation Kerr rotation angle than NdCo. This is in turn because the remanent Kerr rotation angle of these trilayer films is determined by the weighted average of the saturation Kerr rotation angle, the magnetization component perpendicular to a film surface, and the thickness of each layer. The reflectivity of the $\mathrm{NdFeCo}$ trilayer film (T-NdFeCo) decreases monotonically as the wavelength decreases, and shows a slightly higher value than that of the other films throughout the measured range.

The figure of merit, $\sqrt{\bar{R}} \theta_{k}$, for the same samples, calculated from the data in Fig. 4, is plotted as a function of wavelengths in Fig. 5. For comparison, the figures of merit of $\mathrm{Pt} / \mathrm{Co}$ multilayer films are also shown in Fig. 5. $[10,11]$. The value of $\sqrt{R} \theta_{k}$, which is proportional to a readout signal in M.O. recording, is higher in the trilayer films than in $\mathrm{TbFeCo}$ and in the trilayer film with NdCo throughout the measured wavelength range, $\lambda=400-850 \mathrm{~nm}$. Furthermore, the figure of

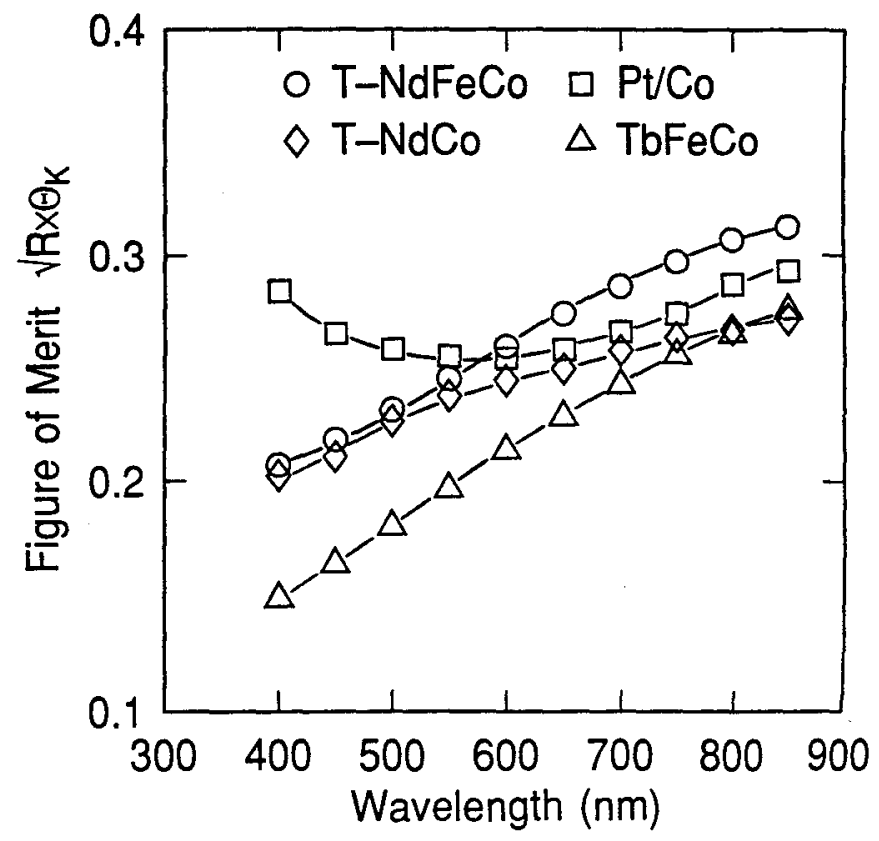

Fig. 5 Light wavelength dependence of figure of merit $\sqrt{\mathrm{R}} \theta_{\mathrm{Kr}}$ 


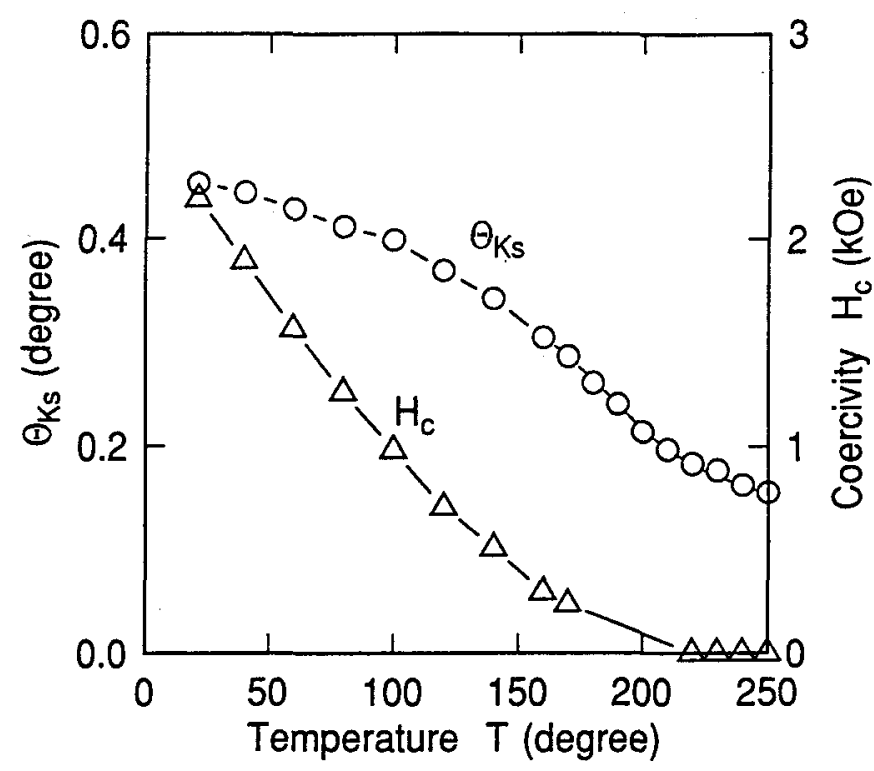

Fig. 6 Temperature dependence of saturation Kerr rotation angle $\theta_{\mathrm{Ks}}$ and coercivity $H_{\mathrm{c}}$ in T-NdFeCo sample.

merit in the present trilayer film is larger than in $\mathrm{Pt} / \mathrm{Co}$ multilayers above $600 \mathrm{~nm}$.

Figure 6 shows the representative temperature dependence of $\theta_{\mathrm{Ks}}$ and $\mathrm{H}_{\mathrm{c}}$ for the $\mathrm{NdFeCo}$ trilayer film (T-NdFeCo) sample. These data are taken from the Kerr loops, measured at a wavelength of $830 \mathrm{~nm}$. Note that the coercivity $H_{c}$ decreases much faster than $\theta_{\mathrm{Ks}}$ and reaches almost zero value at temperatures of around $220^{\circ} \mathrm{C}$. This indicates that the coercive force of $\mathrm{NdFeCo}$ trilayer films is controlled by the coercivity of the top and bottom $\mathrm{TbFeCo}$ layers. The saturation Kerr rotation angle above $220^{\circ} \mathrm{C}$ is determined mainly by the contribution of the second ( $\mathrm{NdFeCo}$ ) layer, because $\mathrm{NdFeCo}$ has a higher Curie temperature than $\mathrm{TbFeCo}$. This tendency is quite similar to that of NdCo trilayer films [9]. In view of these results, $\mathrm{NdFeCo}$ trilayer films are also expected to have a potential for high-density magneto-optical recording at short wavelengths, as well as the NdCo trilayer films reported previously [9].

\section{CONCLUSION}

We investigated the effect on the magnetic and magneto-optical effects of trilayer films of using $\mathrm{NdFeCo}$ for the second layers. A trilayer film, $\mathrm{Tb}_{20} \mathrm{Fe}_{74} \mathrm{Co}_{6}$ $(10 \mathrm{~nm}) / \mathrm{Nd}_{16} \mathrm{Fe}_{57} \mathrm{Co}_{27}(7.5 \mathrm{~nm}) / \mathrm{Tb}_{20} \mathrm{Fe}_{74} \mathrm{Co}_{6}(100 \mathrm{~nm})$, was obtained, with remanent Kerr rotation angle $\theta_{\mathrm{Kr}}=0.32^{\circ}$ at $\lambda=400 \mathrm{~nm}$, coercive force $\mathrm{H}_{\mathrm{c}}=2.4 \mathrm{kOe}$, and a good square loop. The figure of merit $\left(\sqrt{\mathrm{R}} \theta_{\mathrm{k}}\right)$ of this trilayer film is 0.21 at a wavelength of $400 \mathrm{~nm}$.

\section{REFERENCES}

[1] R. J. Gambino and T. R. McGuire, J. Appl. Phys. 57(1), 3906 (1985).

[2] R. J. Gambino and T. R. McGuire, J. Magn. Magn. Mat. 54-57, 1365 (1986).

[3] H. Tanaka and S. Takayama, J. Appl. Phys. 67, 5334 (1990).

[4] S. Tsunashima, Y. J. Choe, S. Tsunashima, T. Katayama, and S. Uchiyama, J. Magn. Soc. Jpn., 11, Suppl. 273 (1987).

[5] R. J. Gambino, T. S. Plaskett, and R. R. Ruf, IEEE Trans. Magn. MAG-24, 2557 (1988).

[6] T. Suzuki and T. Katayama, IEEE Trans. Magn. MAG-22, 1230 (1986).

[7] H. Iiyori and S. Takayama, J. Appl. Phys. 69, 4761 (1991).

[8] H. liyori and S. Takayama J. Mag. Soc. Jpn. 15, supplement, No. S1, 189 (1991).

[9] H. Miyajima, K. Sato, and T. mizoguchi, J. Appl. Phys. 47, 4669 (1976).

[10] S.Hashimoto, Y. Ochiai, and K. Aso, Jpn. J. Appl. Phys. 28, 1824 (1989).

[11] W.B. Zeper, F.J.A.M. Greidanus, and P.F. Carcia, IEEE Trans. Magn. MAG-25, 3764 (1989).

[12] H. Iiyori and S. Takayama, To be published elsewhere. 